\title{
Myogenic Differentiation from MYOGENIN-Mutated Human iPS Cells by CRISPR/Cas9
}

\author{
Koki Higashioka, ${ }^{1,2}$ Noriko Koizumi, ${ }^{2}$ Hidetoshi Sakurai, ${ }^{3}$ Chie Sotozono, ${ }^{1}$ and \\ Takahiko Sato ${ }^{1}$ \\ ${ }^{1}$ Department of Ophthalmology, Kyoto Prefectural University of Medicine, Kyoto, Japan \\ ${ }^{2}$ Department of Biomedical Engineering, Faculty of Life and Medical Sciences, Doshisha University, Kyotanabe, Japan \\ ${ }^{3}$ Department of Clinical Application, Center for iPS Cell Research and Application, Kyoto University, Kyoto, Japan \\ Correspondence should be addressed to Takahiko Sato; takahiko@koto.kpu-m.ac.jp
}

Received 12 January 2017; Accepted 5 March 2017; Published 4 April 2017

Academic Editor: Yuko Miyagoe-Suzuki

Copyright (c) 2017 Koki Higashioka et al. This is an open access article distributed under the Creative Commons Attribution License, which permits unrestricted use, distribution, and reproduction in any medium, provided the original work is properly cited.

\begin{abstract}
It is well known that myogenic regulatory factors encoded by the Myod 1 family of genes have pivotal roles in myogenesis, with partially overlapping functions, as demonstrated for the mouse embryo. Myogenin-mutant mice, however, exhibit severe myogenic defects without compensation by other myogenic factors. MYOGENIN might be expected to have an analogous function in human myogenic cells. To verify this hypothesis, we generated MYOGENIN-mutated human iPS cells by using CRISPR/Cas9 genome-editing technology. Our results suggest that MYOD1-independent or MYOD1-dependent mechanisms can compensate for the loss of MYOGENIN and that these mechanisms are likely to be crucial for regulating skeletal muscle differentiation and formation.
\end{abstract}

\section{Introduction}

In vertebrate embryos, skeletal muscles of the trunk and limbs are derived from the somites, from the dermomyotome which gives rise to myogenic progenitor cells that are directed into the skeletal muscle programme by four myogenic bHLH transcription factors, Myf5, Myod1, Mrf4, and Myogenin (Myog) [1-3]. The myogenic differentiation process in vertebrate embryos is regulated by these factors leading to the formation of multinucleated myotubes and subsequently to the regeneration of skeletal muscle by a reserve of myogenic stem cells in adulthood $[4,5]$.

Single or compound knockout mice for the genes encoding these myogenic factors have been created to identify their function in myogenesis [3]. Single Myf5- or Myod1-deficient mice revealed no striking skeletal muscle phenotype [6-8], pointing to overlapping functions between these myogenic determination factors [9-11]. Mrf4, which is coexpressed with $M y f 5$ at the onset of myogenesis, also acts as an early myogenic determination factor [11]. Double and triple mutants for these genes demonstrate their role in determining muscle cell fate. The fourth member of this gene family, Myog, is expressed at the onset of muscle cell differentiation. Single Myog-deficient mice exhibit severe defects of skeletal muscle formation during development, at a stage when Myod1 and, in many muscles, Mrf4 are also present. This therefore demonstrates that Myog is required for embryonic muscle differentiation, and no redundant or compensatory mechanisms replace its function, unlike for the other myogenic regulatory factors $[10,12-15]$.

In this study, we have tackled the question of whether these myogenic factors have analogous interrelationships in human myogenesis and, in particular, whether human MYOGENIN (MYOG) is also essential for muscle cell differentiation and muscle fiber formation in myogenic cells derived from human induced pluripotent stem (hiPS) cells. To perform functional experiments, we have used versatile genome-editing technology, with the CRISPR/Cas9 system $[16,17]$. 
TABLE 1: Candidates and oligos for MYOGENIN exon1 target positions for CRISPR/Cas9.

\begin{tabular}{|c|c|c|c|c|c|c|c|}
\hline \multirow{2}{*}{$\begin{array}{l}\text { Position } \\
\text { Start-end }\end{array}$} & \multirow[b]{2}{*}{$+1-$} & \multirow{2}{*}{$\begin{array}{c}\text { Target sequence } \\
20 \mathrm{bp}+\mathbf{P A M}\end{array}$} & \multicolumn{2}{|c|}{ Sequence information } & \multicolumn{3}{|c|}{ Number of target sites } \\
\hline & & & GC (\%) & $\operatorname{Tm}\left({ }^{\circ} \mathrm{C}\right)$ & $20 \mathrm{bp}$ & $12 \mathrm{bp}$ & $8 \mathrm{bp}$ \\
\hline $143-165$ & - & cctgcctgtccacctccagggct & 70 & 84 & 1 & 61 & 5171 \\
\hline $147-169$ & - & cctgtccacctccagggcttcga & 65 & 80 & 1 & 38 & 92,481 \\
\hline $152-174$ & - & ccacctccagggcttcgaaccac & 65 & 79 & 1 & 89 & 10,513 \\
\hline $155-177$ & - & cctccagggcttcgaaccaccag & 65 & 79 & 1 & 8 & 11,331 \\
\hline $156-178$ & + & ctccagggcttcgaaccaccagg & 65 & 79 & 1 & 1 & 4654 \\
\hline $158-180$ & - & ccagggcttcgaaccaccaggct & 65 & 81 & 1 & 7 & 21,861 \\
\hline $166-188$ & + & tcgaaccaccaggctacgagcgg & 60 & 77 & 1 & 10 & 12,349 \\
\hline $170-192$ & + & accaccaggctacgagcggacgg & 65 & 82 & 1 & 1 & 3812 \\
\hline $171-193$ & - & ccaccaggctacgagcggacgga & 70 & 82 & 1 & 8 & 1537 \\
\hline $174-196$ & - & ccaggctacgagcggacggagct & 70 & 83 & 1 & 3 & 479 \\
\hline $191-213$ & + & ggagctcaccctgagccccgagg & 75 & 84 & 1 & 43 & 382 \\
\hline
\end{tabular}

pX458-hMYOG+189_F primer: CACCaccaccaggctacgagcgga. pX458-hMYOG+189_R primer: AAACtccgctcgtagcctggtggt.

\section{Materials and Methods}

2.1. Gene Targeting with Human iPS Cells. The hMYOGtargeting plasmid vector, pX458-hMYOG+189, was constructed using the pX458 vector (Addgene \#48138, Cambridge, USA) [17] with ligating oligos (Table 1) as described, and plasmid DNA was introduced into HEK293- or Hu5/KD3immortalized human myogenic cells [18], with ViaFect reagent (Promega, Madison, USA). The electroporator NEPA21 (NEPA GENE, Chiba, Japan) was used for introducing plasmids into hiPS cells [19].

2.2. Cell Culture and Myogenic Differentiation. The hiPS cells were maintained on SNL feeder cells, treated with $10 \mu \mathrm{g} / \mathrm{ml}$ of mitomycin (Sigma, St. Louis, USA) in DMEM (Wako, Osaka, Japan) supplemented with $10 \%$ of fetal bovine serum (GIBCO, Grand Island, USA), or expanded in Primate ES cell medium (ReproCELL, Kanagawa, Japan) supplemented with $10 \mathrm{ng} / \mathrm{ml}$ of recombinant human FGF2 (bFGF; Wako, Osaka, Japan) and $100 \mu \mathrm{g} / \mathrm{ml}$ of G418 (Nacalai Tesque, Kyoto, Japan). MYOG-deficient iPS cells were maintained on SNL feeder cells or iMatrix-511 (Nippi, Tokyo, Japan)coated plates with StemFit AK03N (Ajinomoto, Tokyo, Japan) under a feeder-free culture system [20].

For the derivation of myogenic cells from hiPS cells, the detailed protocol of Tanaka et al., based on MYOD1 induction [21], was followed. In brief, single iPS cells carrying an inducible MYOD1 activation system were expanded in Primate ES cell medium without bFGF and with $10 \mu \mathrm{M}$ of Y-27632 (Nacalai Tesque, Kyoto, Japan) for 24 hours and then induced into myogenic cells by adding $500 \mathrm{ng} / \mathrm{ml}$ of doxycycline (Dox; Tocris, Bristol, UK). After 24 hours, culture medium was changed into myogenic differentiation medium composed of alpha-MEM (Nacalai Tesque, Kyoto, Japan) with $5 \%$ of KSR (GIBCO, Grand Island, USA) and $500 \mathrm{ng} / \mathrm{ml}$ of Dox. After 6 days, culture medium was changed into muscle maturation medium, DMEM/F12 (Nacalai Tesque, Kyoto, Japan), with $5 \%$ of horse serum (Sigma, St. Louis, USA), $10 \mathrm{ng} / \mathrm{ml}$ of recombinant human insulinlike growth factor 1 (IGF-1; PeproTech, Hartford County,
USA), and $200 \mu \mathrm{M}$ of 2-mercaptoethanol (2-ME; Sigma, St. Louis, USA).

To obtain myogenic cells derived from embryonic mesodermal cells, single iPS cells were expanded in StemFit AK03N supplemented with $10 \mu \mathrm{M}$ of Y-27632. After 2 days, the culture medium was changed into modified mesodermal differentiation medium as described by Loh et al. [22]. Cultured cells were passaged 12 days later and cultured in mesoderm differentiation medium with $10 \mu \mathrm{M}$ of $\mathrm{Y}-27632$ for 2 days. To initiate myogenic differentiation, medium SF-O3 (EIDIA, Tokyo, Japan), supplemented with $10 \mathrm{ng} / \mathrm{ml}$ of bFGF, $10 \mathrm{ng} / \mathrm{ml}$ of IGF-1, $10 \mathrm{ng} / \mathrm{ml}$ of HGF (PeproTech, Hartford, USA), and $200 \mu \mathrm{M}$ of $2-\mathrm{ME}$, was used and changed into myogenic differentiation medium with IGF-1 after 4 days and then with IGF-1 and HGF after 3 days [23]. To obtain more mature myogenic differentiation, culture medium was changed into DMEM/F12 supplemented with $2 \%$ of horse serum, $10 \mathrm{ng} / \mathrm{ml}$ of IGF-1, and $200 \mu \mathrm{M}$ of 2-ME 2 weeks later and induced cells were harvested at day $60[23]$.

2.3. Cell Sorting. Cultured cells transfected with pX458hMYOG+189 were dissociated with TrypLE select (GIBCO, Grand Island, USA) at $37^{\circ} \mathrm{C}$ for $5 \mathrm{~min}$ for detecting transfected cells. Dissociated cells were resuspended with $1 \%$ bovine serum albumin in PBS. Cell debris were eliminated with a cell strainer ( $35 \mu \mathrm{m}$; BD, New Jersey, USA), and suspensions were stained with propidium iodide (Molecular Probes, Eugene, USA) to exclude dead cells. Cells were analyzed and collected by a cell sorter using FACSJazz (BD, New Jersey, USA).

2.4. Quantitative PCR Analyses. Total RNAs from sorted or cultured cells were extracted using the RNeasy micro kit (QIAGEN, Hilden, Germany). For quantitative PCR analyses, single strand cDNA was prepared using a SuperScript VILO kit (Invitrogen, Carlsbad, USA) as in the manufacturer's protocol. All RT-qPCR reactions were carried out in triplicate using THUNDERBIRD SYBR qPCR Mix (TOYOBO, Osaka, Japan), normalized to the mRNA expression level of 
TABLE 2: Primer sequences for T7 endonuclease assay and quantitative RT-PCR.

\begin{tabular}{|c|c|c|}
\hline Genes & Sequences & Amplicon size \\
\hline \multicolumn{3}{|l|}{ Primer for T7 EI assay } \\
\hline MYOG gDNA_F & 5'-GGCCGCCCAGCTAGGAGTAATTGA-3' & \multirow[b]{2}{*}{786} \\
\hline MYOG exon1_R & 5'CGCTCGATGTACTGGATGGCACTG-3' & \\
\hline \multicolumn{3}{|l|}{ Primer for RT-qPCR } \\
\hline RPL13A_F & 5'-CCCTGGAGGAGAAGAGGAAA-3' & \multirow[b]{2}{*}{91} \\
\hline RPL13A_R & 5'-ACGTTCTTCTCGGCCTGTTT-3' & \\
\hline$M Y O G \_F$ & 5'-GCTCAGCTCCCTCAACCA-3' & \multirow{2}{*}{94} \\
\hline$M Y O G \_\mathrm{R}$ & 5'-GCTGTGAGAGCTGCATTCG-3' & \\
\hline MYOD1_F & 5'-GCACTACAGCGGCGACTCC-3' & \multirow{2}{*}{118} \\
\hline MYOD1_R & 5'-GTAGGCGCCTTCGTAGCAG-3' & \\
\hline Endo-MYOD1_F & 5'-CACTCCGGTCCCAAATGTAG-3' & \multirow{2}{*}{180} \\
\hline Endo-MYOD1_R & 5'-TTCCCTGTAGCACCACACAC-3' & \\
\hline MRF4_F & 5'-GGCCAAGTGTTTCCGATCAT-3' & \multirow{2}{*}{89} \\
\hline MRF4_R & 5'AAGGCTACTCGAGGCTGACG-3' & \\
\hline
\end{tabular}

ribosomal protein L13A (RPL13A). Primer sequences ( $5^{\prime}$ to $3^{\prime}$ ) are listed in Table 2.

2.5. Immunofluorescence Assay. Cultured cells were fixed in $4 \%$ paraformaldehyde for $10 \mathrm{~min}$ at $4^{\circ} \mathrm{C}$, permeabilized with $0.2 \%$ Triton and $50 \mathrm{mM} \mathrm{NH}_{4} \mathrm{Cl}$. Fixed samples were pretreated with Blocking One (Nacalai Tesque, Kyoto, Japan) for $30 \mathrm{~min}$ at $\mathrm{RT}$ and incubated with anti-MYOGENIN (diluted 1:100, Santa Cruz Biotechnology, California, USA), anti-MYOSIN HEAVY CHAIN (MYHC, diluted 1:200, Santa Cruz Biotechnology, California, USA), anti-TRA-1-81 (diluted 1:200, Cell Signaling Technology, Massachusetts, USA), anti-SSEA4 (diluted $1: 200$, Cell Signaling Technology, Massachusetts, USA), anti-OCT4A (diluted 1:200, Cell Signaling Technology, Massachusetts, USA), and anti-NANOG (diluted 1:200, Cell Signaling Technology, Massachusetts, USA) antibodies in 5\% of Blocking One in PBS with $0.1 \%$ Tween 20 (PBST) overnight at $4^{\circ} \mathrm{C}$. After three washes with PBST, cells were incubated with Alexa488-, Alexa594-, or Alexa647-conjugated secondary antibodies (diluted 1:500, Molecular Probes, Eugene, USA). Cells were washed with PBST three times and mounted in SlowFade Diamond antifade mountant with DAPI (Molecular Probes, Eugene, USA). Fluorescent images were collected on the software of BZ-X700 (Keyence, Osaka, Japan). Cultured cells were analyzed from triplicate experiments.

\section{Results}

3.1. Human MYOGENIN Genomic DNA Editing with the CRISPR/Cas9 System. To generate MYOG-mutated hiPS cells by double-strand break in MYOG exon 1 which includes coding sequence (Figure 1(a)), we selected several sequences bound to single guide RNA for targeting by nuclease Cas9 from the CRISPRdirect website as candidates (http://crispr. dbcls.jp, Table 1) [24] and ligated them into the pX458 vector to create the pX458-hMYOG+189-editing vector, which targets a unique $20 \mathrm{bp}$ sequence in $h M Y O G$ exon 1 (position 170-192; accaccaggctacgagcgga, Figure 1(b)). The effect of a double-strand break in $h M Y O G$ genomic sequences was evaluated by heteroduplex PCR fragments, involving the sequences targeted by the pX458-hMYOG+ 189-editing vector, monitored in HEK293 cells and T7 endonuclease I (T7EI). Enzymatic digested PCR bands of $500 \mathrm{bp}$ and $300 \mathrm{bp}$ were observed in T7EI-treated genomic DNA (Figure 1(c)). The data suggested that nuclease Cas9 and single guide RNA target $h M Y O G$ genomic sequences of exon1. The expression of $M Y O G$ is initiated in differentiating myogenic cells. To check the amount of MYOG transcripts produced from this Cas9 construct, immortalized Hu5/KD3, human myoblasts, transfected with or without the pX458-hMYOG+ 189 vector were differentiated in medium with $2 \%$ horse serum for 48 hours. The transcriptional level of MYOG was attenuated in differentiated Hu5/KD3 cells (Figure 1(d)). This CRISPR/Cas9 construct for $h M Y O G$ sequences may not only be effective because of its genomic double-strand break which knocks out MYOG expression but may also affect the remaining $M Y O G$ transcription level.

3.2. Generation of MYOGENIN-Mutated hiPS Cells. In order to generate MYOG-mutated hiPS cells, we used hiPS cells carrying a MYOD1 expression construct which is inducible with Dox to activate the myogenic programme (Figure 2(a)) [21]. The iPS cells were expanded on SNL feeder-coated plates after electroporation with pX458$h M Y O G+189$ vector for 48 hours, and GFP-positive cells were collected by cell sorting (Figures 2(b) and 2(c)). These cells were plated out to form colonies which were individually picked up. Each clone was screened for further analyses.

We were able to identify 25 clones, which were lacking the wild-type MYOG sequences (wild type: $19.4 \%$, heterozygotes; $64.5 \%$, homozygotes; and $16.1 \%$, total screened clones $n=31$ ) by checking genomic sequences around the targeted MYOG region. Selected clone number 28 or clone number C3 was confirmed to have biallelic on-target frameshift 


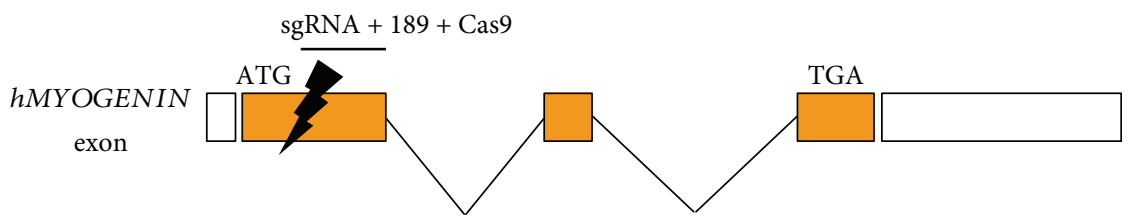

(a)

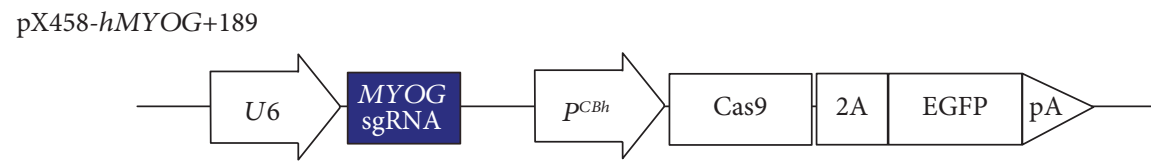

(b)

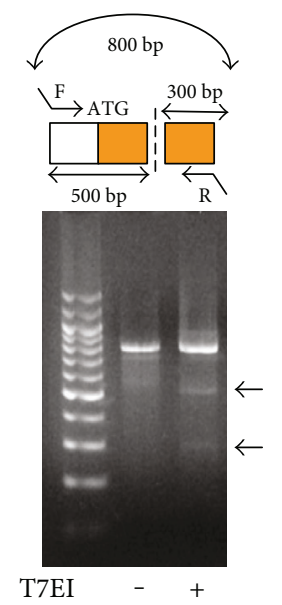

(c)

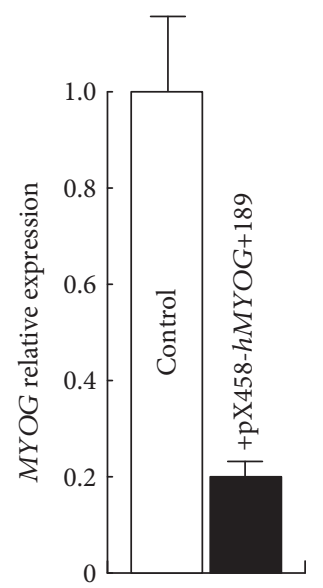

(d)

FIgURE 1: Effect of single guide sequence for $h M Y O G E N I N$ by the CRISPR/Cas9 system. A schematic representation of $M Y O G$ exons and introns. A candidate position for Cas9 targeting of $M Y O G$ exon1 (a). pX458- $h M Y O G+189$, a construct for driving single guide RNA bound to MYOG exon1 and bicistronic expression of both Cas9 and GFP (b). T7 endonuclease I assay for Cas9-mediated cleavage (arrows, $500 \mathrm{bp}$ and $300 \mathrm{bp}$ ) on an agarose gel, showing comparable modification of the targeted human MYOG genomic fragment in HEK293T cells (c). Relative expression of MYOG in Hu5-immortalized human myoblast cells transfected with or without the pX458$h M Y O G+189$ vector. All error bars indicate $\pm \operatorname{SEM}(n=3)$.

mutations, $5 \mathrm{bp}$ of deletion, and an extra $1 \mathrm{bp}$ of integration in the $h M Y O G$-sgRNA and Cas9-targeted region as shown in Figure 2(d). These data suggest that this targeting CRISPR/Cas9 system is sufficiently efficient to knockout both alleles of MYOG directly by introducing out-of-frame mutations (lower images in Figure 2(f)). MYOG-mutated hiPS cells (clone number 28) were immunostained with undifferentiated pluripotent markers, anti-SSEA4, antiOCT3/4, anti-TRA1-80, and anti-NANOG antibodies, to evaluate the undifferentiated pluripotent state, and these markers were detected positively in MYOG-mutated hiPS cells (Figure 2(e)). To confirm the translation of truncated MYOG protein from these mutated sequences, myogenic cells differentiated from Dox-treated hiPS cells for 7 days were immunoreacted with antibodies against human MYOG N-terminus and C-terminus relatively because $M Y O G$ mRNAs are transcribed with the extra stop codon, which results from the MYOG gene targeting. Myogenic cells derived from wild-type hiPS cells were detected by both of these MYOG antibodies; however, the C-terminus of
MYOG was not detected in MYOG-mutated hiPS cells (Figure 2(f)).

3.3. Skeletal Myogenic Differentiation by MYOD1 Induction. To investigate human MYOG function during myogenic differentiation, MYOD1 was overexpressed in hiPS cells by administrating Dox as shown in Figure 3(a). MYOD1 expression mimics bicistronic mCherry fluorescence after Dox treatment (Figure 3(b)). Induced myogenic cells derived from hiPS cells were cultured in vitro under differentiation conditions and immunostained for MYHC expression as an indicator of their ability to differentiate into skeletal muscle fibers (Figure 3(c)). Although the rate of myoblast fusion in $M Y O G$-mutated hiPS cell clone number 28 was slightly less than that of wild type (Figure $3(\mathrm{~d})$ ), terminal differentiation is similar.

To further characterize the differentiation of these myogenic cells, RNA expression of myogenic factors was analyzed by quantitative RT-PCR. The transcript for MYOG was downregulated as shown in Figure 1(d) with unknown mechanisms; however, other myogenic factors, notably 


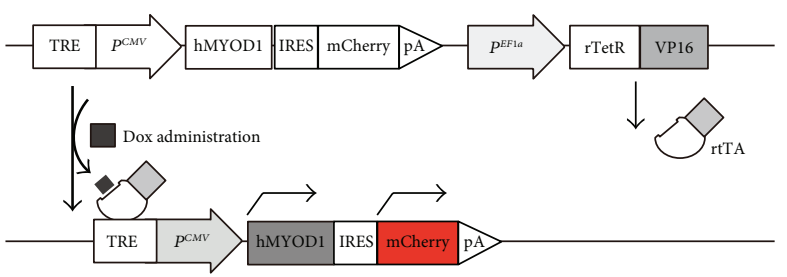

(a)

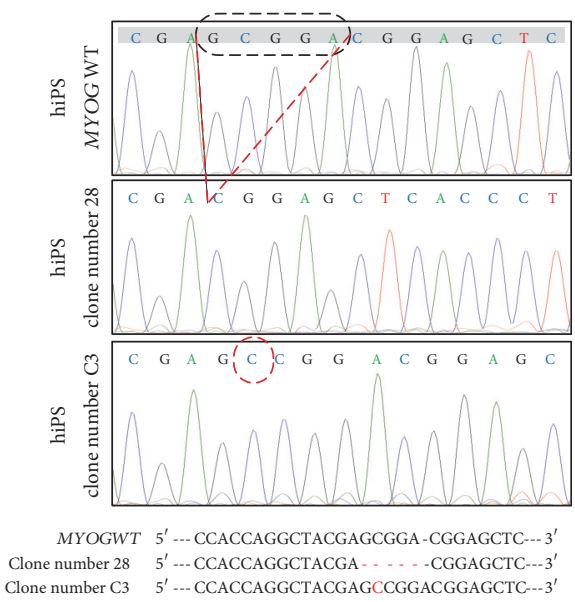

(d)

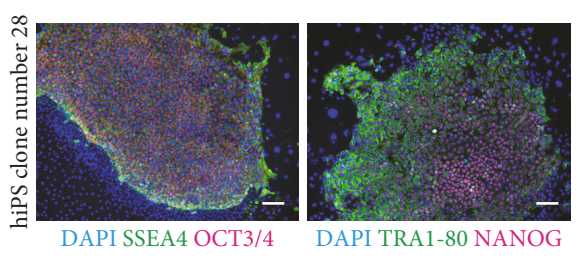

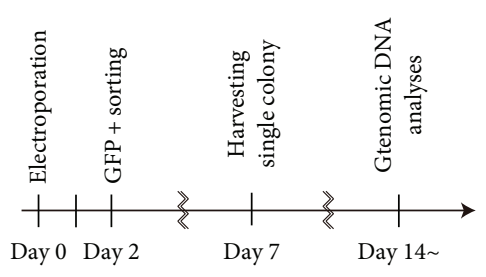

(b)

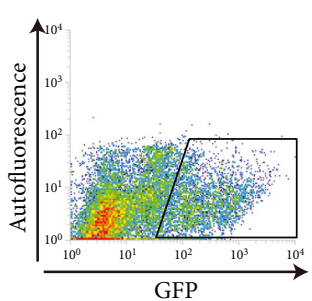

(c)
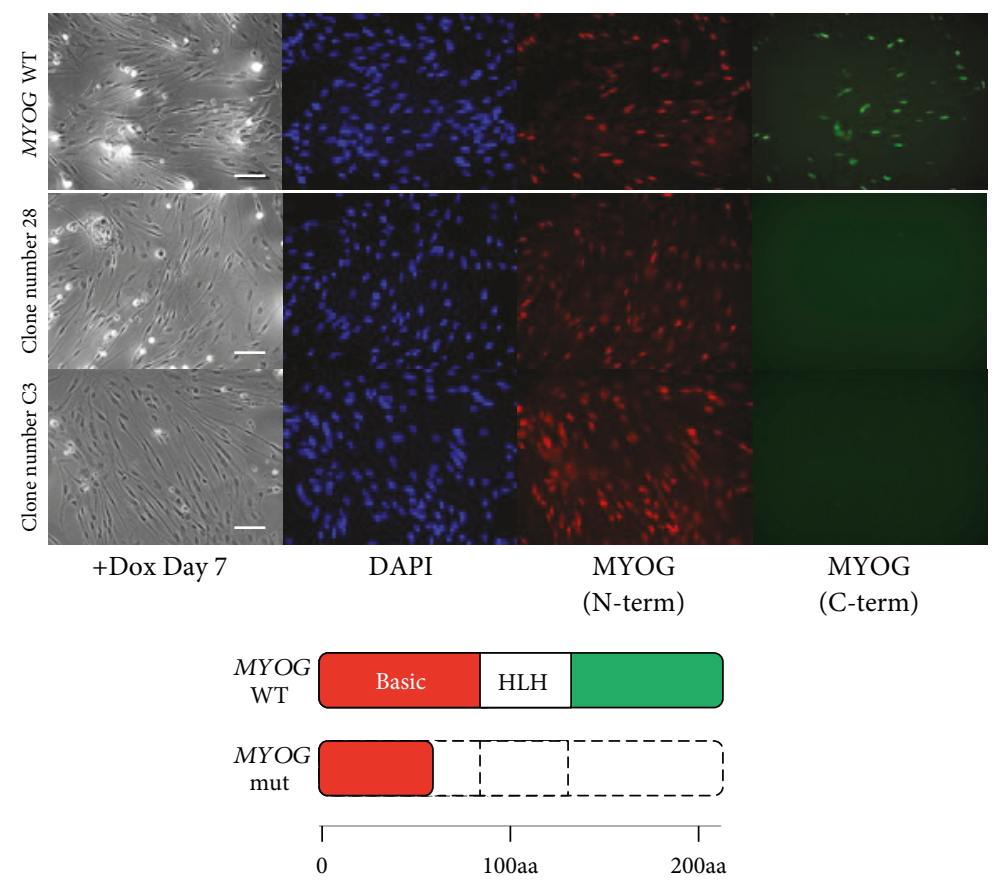

(f)

(e)

FIGURE 2: Generation of $h M Y O G E N I N$-mutated hiPS cells. A schematic model for the induction of myogenic cells derived from hiPS cells by overexpression of MYOD1 marked with mCherry (red) after administrating Dox (a). A flowchart of the time course for the identification of MYOG-mutated hiPS cells (b). FACS analyses to isolate hiPS cells after the introduction of the pX458-hMYOG+189 vector (c). Genomic sequence data around the region targeted by pX458-hMYOG+189. $5 \mathrm{bp}$ of deletion in clone number 28 and 1 bp of insertion in clone number C3 (dashed lines, (d)). Established hiPS cells were immunostained with undifferentiated pluripotent cell markers, anti-SSEA4 (green in left panel), anti-OCT3/4 (red in left panel), anti-TRA1-80 (green in right panel), and anti-NANOG (red in right panel) antibodies. Nuclei were stained with 4'6-diamidino-2-phenylindole (DAPI, blue). Scale bar, $100 \mu \mathrm{m}$ (e). Differentiated myogenic cells after Dox treatment for 7 days were immunostained with anti-MYOG N-terminus (N-term, red) and C-terminus (C-term, green) antibodies. Nuclei were stained with $4^{\prime} 6$-diamidino-2-phenylindole (DAPI, blue). Scale bar, $100 \mu \mathrm{m}$ (upper in (f)). Putative MYOG protein structures both in wild-type (MYOG WT) and mutated cells (MYOG mut) (lower in (f)).

transcripts of $M Y O D 1$ or $M R F 4$, were upregulated under conditions where MYOG is mutated in human myogenic cells (Figures 3(e)-3(g)).

3.4. Skeletal Muscle Differentiation via Mesodermal Differentiation In Vitro. Transient overexpression of MYOD1 might have overcome the effect of MYOG deficiency because artificially high MYOD1 may compensate the inactivation of the MYOG gene in human myogenic cells. To avoid excessive MYOD1 levels, myogenic cells were induced from mesodermal precursors derived from hiPS cell clone number 28, without administration of Dox as shown in Figure 4(a).

The percentage of mesodermal induction marked by DLL1 [22] was shown by FACS analyses and was similar irrespective of MYOG mutation (Figure 4(b)). In myogenic cells derived from mesodermal precursors, total MYOD1 transcripts did not accumulate, in contrast to Dox-treated hiPS cells, including lower level of endogenous MYOD1 expression (Figure 4(c)). Under these conditions, MYHCpositive differentiated myofibers derived from both MYOGpositive and MYOG-negative hiPS cells were identified to a similar extent (Figure 4(d)). To analyze myogenic differentiation potential from mesodermal cells, transcripts of myogenic regulatory factors were monitored in these cells. The level of MYOG transcript was attenuated; however, MYOD1 or $M R F 4$ transcripts were not much changed in wild-type and MYOG-mutated myogenic cells, as upregulated in MYOGmutated cells during periods of cell culture (Figure 4(e)). 

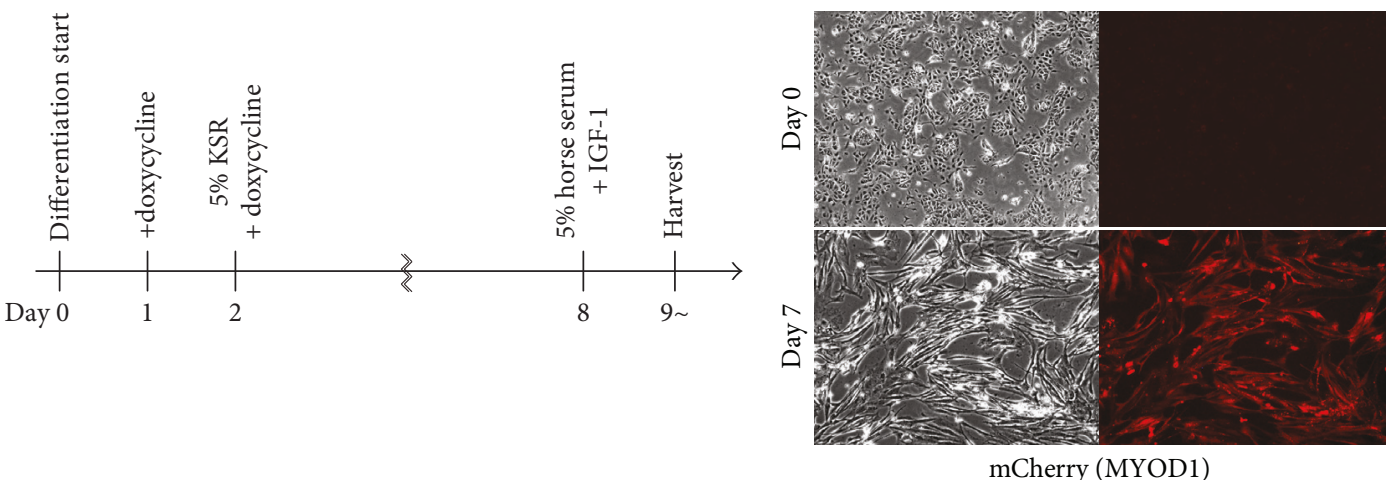

(a)

(b)

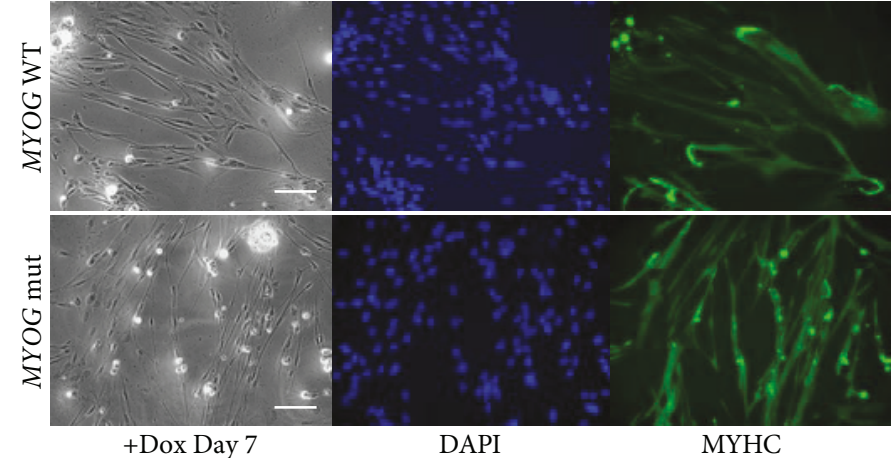

(c)

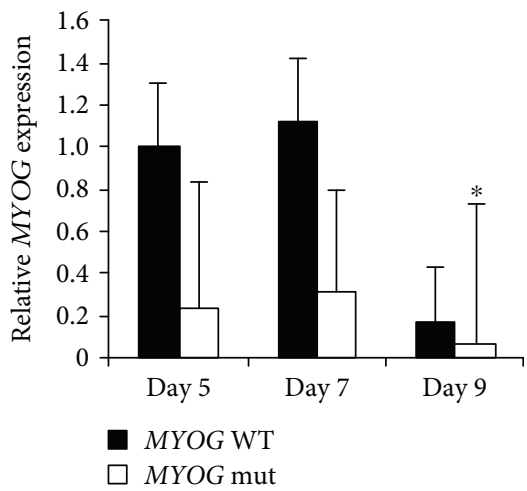

(e)

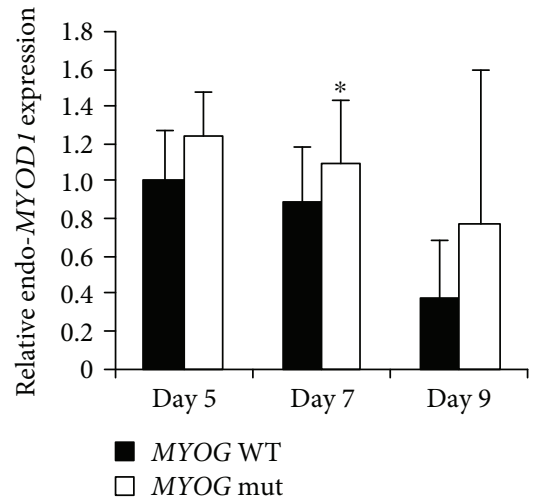

(f)

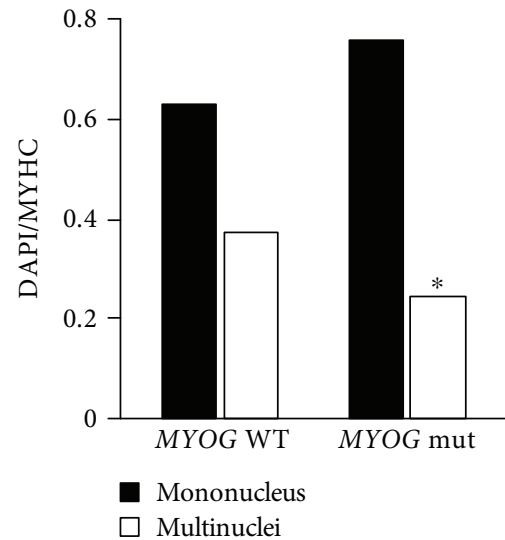

(d)

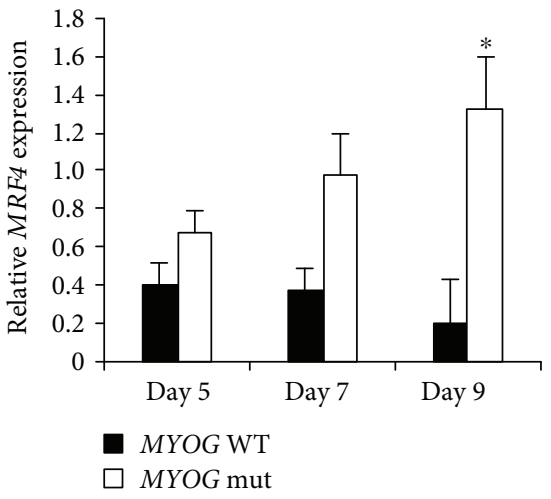

(g)

FIGURE 3: Myogenic differentiation in skeletal muscle cells derived from MYOGENIN-deficient hiPS clone number 28 cells. Myogenic differentiation flowchart of the time course (a). Morphological changes and mCherry fluorescent expression after treatment with Dox (b). Differentiated myogenic cells derived from hiPS cells with or without MYOG by Dox treatment for 7 days were immunostained with anti-MYOSIN HEAVY CHAIN (MYHC, green) antibody. Nuclei were stained with 4'6-diamidino-2-phenylindole (DAPI, blue). Scale bar, $100 \mu \mathrm{m}$ (c). The ratio of DAPI-positive mono or multiple nuclei staining present in single MYHC-positive myofibers derived from wild-type or MYOG-mutated hiPS cells (d). Relative expression of transcripts for myogenic regulatory factors, MYOG (e), endogenous MYOD1 (f), and MRF4 (g), in differentiated myogenic cells treated with Dox for 5, 7, and 9 days. All error bars indicate \pm SEM $(n=3)$. $P$ values are determined by a $t$-test from a two-tailed distribution. ${ }^{*} P<0.05$.

\section{Discussion}

Here, we report the generation of MYOGENIN-deficient hiPS cells and the impact on human myogenic differentiation using CRISPR/Cas9 technology. This bacterial system has emerged as an effective tool for gene targeting through nonhomologous end joining (NHEJ); however, it has been reported to be inefficient for precise editing of genomic sequences. In this study, we selected the sequence of $M Y O G$ exon1-targeted sgRNA with the Cas9 complex as a unique 


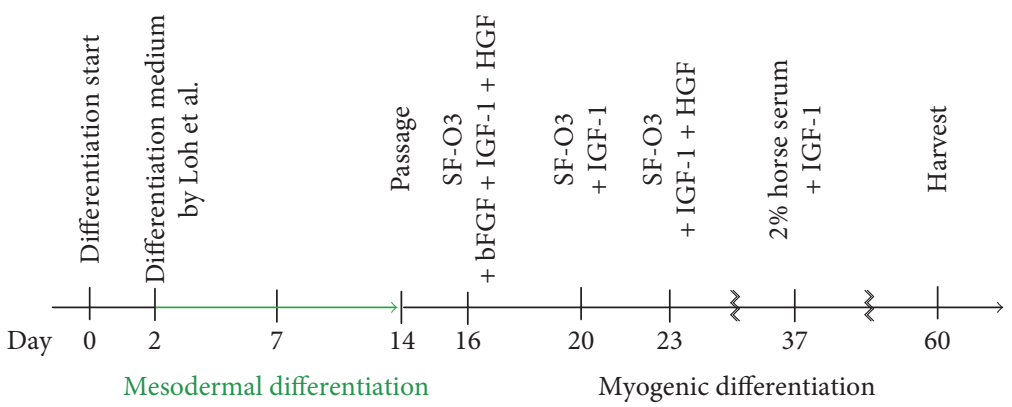

(a)

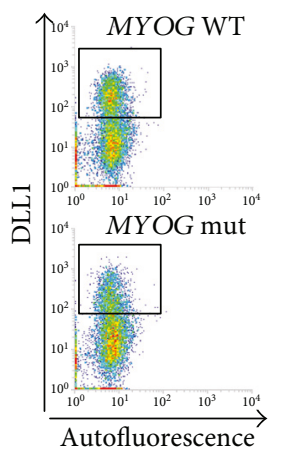

(b)
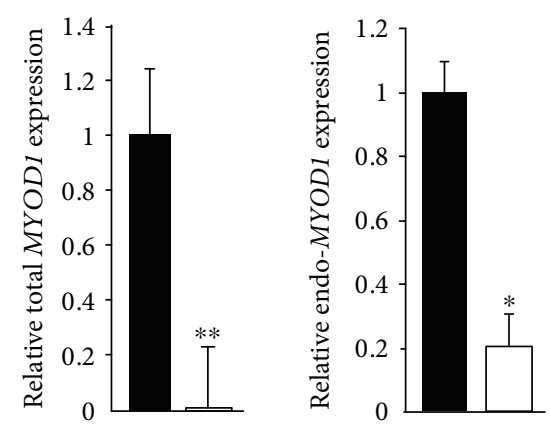

Dox-induced myogenic cells $\square$ Myogenic cells from mesoderm

(c)

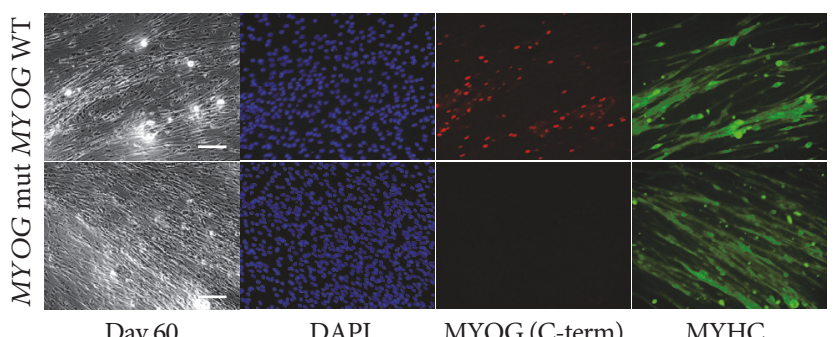

(d)
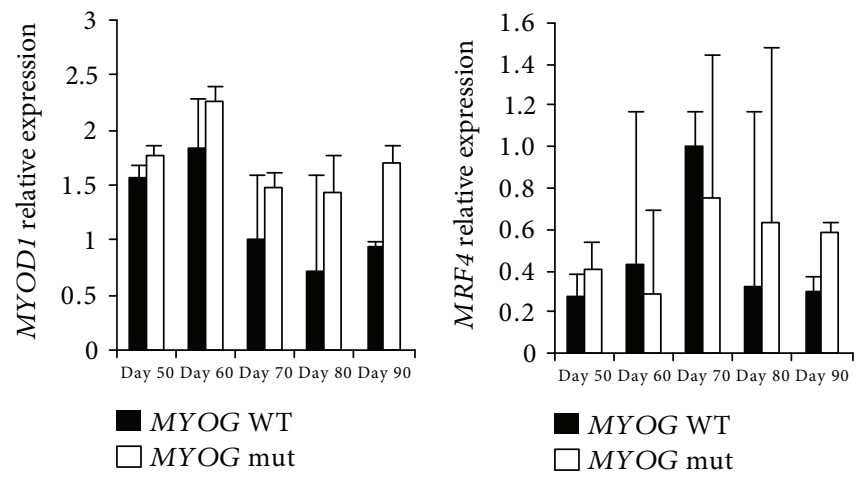

(e)

FIGURE 4: Myogenic differentiation from mesodermal precursors derived from MYOGENIN-deficient hiPS clone number 28 cells. Mesodermal and myogenic differentiation flowchart of the time course for muscle cells derived from hiPS cells (a). FACS analyses of DLL1-positive mesodermal cells derived from hiPS cells (b). Relative expression of total MYOD1 and endogenous MYOD1 (c). Differentiated myogenic cells derived from mesodermal cells with or without MYOG for 60 days were immunostained with anti-MYOSIN HEAVY CHAIN (MYHC, green) antibody. Nuclei were stained with 4'6-diamidino-2-phenylindole (DAPI, blue). Scale bar, $100 \mu \mathrm{m}$ (d). Relative expressions of MYOD1 and MRF4 transcripts in wild-type or MYOG-mutated myogenic cells derived from mesodermal cells (e). All error bars indicate \pm SEM $(n=3)$. $P$ values are determined by a $t$-test from a two-tailed distribution. ${ }^{*} P<0.05,{ }^{* *} P<0.01$. 
in genomic sequence, which targeted MYOG by the T7EI assay not with high efficiency; however, the result of genomic editing in hiPS cells showed high efficiency for knocking out the MYOG gene, including in heterozygotes with an efficiency of over $80 \%$. This was not changed with additional azidothymidine, which has been reported to increase the efficiency for NHEJ [25] (not shown).

While knockout mice of Myog exhibit a lethal deficiency of differentiated skeletal myofibers, there are nevertheless residual myofibers in Myog mutants [12, 13]. The possible differences between in vivo and in vitro situation of $M y o g$ mutants could be explained by the selection of a particular route to muscle cell differentiation from Myog-independent lineage in vitro, potentially controlled by MyoD1 and Mrf4 because Mrf4 can drive early myogenic differentiation in the myotome when Myog protein is not initially accumulated $[10,14]$. Alternatively, there may be a threshold level of total myogenic regulatory factors required in myoblasts to trigger the terminal differentiation program. We have not observed any deficiencies of myogenic differentiation with MYOG-mutated cells under two different conditions, either with overexpression of MYOD1 or through medium conditions that promote mesodermal cell progression towards myogenesis. Mutated hiPS cells without not only MYOG but also other myogenic factors, MYF5, MYOD1, and MRF4, would be necessary for further analyses to identify the relationships of human myogenic regulatory factors because we observed the upregulation of other myogenic factors in MYOG-mutated cells which might compensate MYOG functions in vitro, and triple knockout of Myog, Mrf4, and Myod1 or Myf5, Myod1, and Mrf4 exhibited impaired ability to terminally differentiate into myofibers, not double knockout of Myod1 and Mrf4 [8, 10]. Moreover, there is also other possibility that Myog via skeletal muscle affects systemic factors in vivo [13] and that this feeds back on myofiber formation.

Taken together, these results demonstrate that MYOGmutated human iPS cells have the capacity for myogenic differentiation and can form terminally differentiated myofibers, under differentiation conditions, in contrast to results on developing mouse Myog mutants.

\section{Conflicts of Interest}

No competing financial interests exist.

\section{Acknowledgments}

This research has been supported by grants-in-aid from the Japan Agency for Medical Research and Development, Projects for Technology Development, Development of Cell Transplantation Methods for Refractory Muscle Diseases, and AMED-CREST (AMED) and a grant from Nakatomi Memorial Foundation. The authors are grateful for $\mathrm{Hu} 5 /$ KD3-immortalized human myogenic cells provided by Dr. Naohiro Hashimoto (National Center for Geriatrics and Gerontology, Ōbu, Japan) and for the advice about the maintenance of cell culture from Tomoko Horikiri.

\section{References}

[1] J. Molkentin and E. Olson, "Defining the regulatory networks for muscle development," Current Opinion in Genetics \& Development, vol. 6, no. 4, pp. 445-453, 1996.

[2] M. Buckingham, "Skeletal muscle formation in vertebrates," Current Opinion in Genetics \& Development, vol. 11, no. 4, pp. 440-448, 2001.

[3] M. E. Pownall, M. K. Gustafsson, and C. P. Emerson Jr., "Myogenic regulatory factors and the specification of muscle progenitors in vertebrate embryos," Annual Review of Cell and Developmental Biology, vol. 18, pp. 747-783, 2002.

[4] M. Parker, P. Seale, and M. Rudnicki, "Looking back to the embryo: defining transcriptional networks in adult myogenesis," Nature Reviews Genetics, vol. 4, no. 7, pp. 497-507, 2003.

[5] R. Sambasivan and S. Tajbakhsh, "Skeletal muscle stem cell birth and properties," Seminars in Cell \& Developmental Biology, vol. 18, no. 6, pp. 870-882, 2007.

[6] M. A. Rudnicki, T. Braun, S. Hinuma, and R. Jaenisch, "Inactivation of $M y o D$ in mice leads to up-regulation of the myogenic HLH gene $M y f-5$ and results in apparently normal muscle development," Cell, vol. 71, no. 3, pp. 383-390, 1992.

[7] T. Braun, M. A. Rudnicki, H. Arnold, and R. Jaenisch, "Targeted inactivation of the muscle regulatory gene $M y f-5$ results in abnormal rib development and perinatal death," Cell, vol. 71, no. 3, pp. 369-382, 1992.

[8] B. Kablar, K. Krastel, S. Tajbakhsh, and M. A. Rudnicki, "Myf5 and $M y o D$ activation define independent myogenic compartments during embryonic development," Developmental Biology, vol. 258, no. 2, pp. 307-318, 2003.

[9] A. Rawls, J. Morris, M. Rudnicki et al., "Myogenin's functions do not overlap with those of MyoD or Myf-5 during mouse embryogenesis," Developmental Biology, vol. 172, no. 1, pp. 37-50, 1995.

[10] M. Valdez, J. Richardson, W. Klein, and E. Olson, "Failure of Myf5 to support myogenic differentiation without Myogenin, MyoD, and MRF4," Developmental Biology, vol. 219, no. 2, pp. 287-298, 2000.

[11] L. Kassar-Duchossoy, B. Gayraud-Morel, D. Gomes et al., "Mrf4 determines skeletal muscle identity in Myf5: Myod doublemutant mice," Nature, vol. 431, no. 7007, pp. 466-471, 2004.

[12] P. Hasty, A. Bradley, J. Morris et al., "Muscle deficiency and neonatal death in mice with a targeted mutation in the myogenin gene," Nature, vol. 364, no. 6437, pp. 501-506, 1993.

[13] Y. Nabeshima, K. Hanaoka, M. Hayasaka et al., "Myogenin gene disruption results in perinatal lethality because of severe muscle defect," Nature, vol. 364, no. 6437, pp. 532-535, 1993.

[14] J. Venuti, J. Morris, J. Vivian, E. Olson, and W. Klein, "Myogenin is required for late but not early aspects of myogenesis during mouse development," Journal of Cell Biology, vol. 128, no. 4, pp. 563-576, 1995.

[15] C. Berkes and S. Tapscott, "MyoD and the transcriptional control of myogenesis," Seminars in Cell \& Developmental Biology, vol. 16, no. 4-5, pp. 585-595, 2005.

[16] M. Jinek, K. Chylinski, I. Fonfara, M. Hauer, J. Doudna, and E. Charpentier, "A programmable dual-RNA-guided DNA endonuclease in adaptive bacterial immunity," Science, vol. 337, no. 6096, pp. 816-821, 2012.

[17] L. Cong, F. Ran, D. Cox et al., "Multiplex genome engineering using CRISPR/Cas systems," Science, vol. 339, no. 6121, pp. 819-823, 2013. 
[18] K. Shiomi, T. Kiyono, K. Okamura et al., "CDK4 and cyclin D1 allow human myogenic cells to recapture growth property without compromising differentiation potential," Gene Therapy, vol. 18, no. 9, pp. 857-866, 2011.

[19] H. Li, N. Fujimoto, N. Sasakawa et al., "Precise correction of the dystrophin gene in Duchenne muscular dystrophy patient induced pluripotent stem cells by TALEN and CRISPR-Cas9," Stem Cell Reports, vol. 4, no. 1, pp. 143-154, 2015.

[20] M. Nakagawa, Y. Taniguchi, S. Senda et al., "A novel efficient feeder-free culture system for the derivation of human induced pluripotent stem cells," Scientific Reports, vol. 4, p. 3594, 2014.

[21] A. Tanaka, K. Woltjen, K. Miyake et al., "Efficient and reproducible myogenic differentiation from human iPS cells: prospects for modeling Miyoshi myopathy in vitro," PLoS One, vol. 8, no. 4, article e61540, 2013.

[22] K. Loh, A. Chen, P. Koh et al., "Mapping the pairwise choices leading from pluripotency to human bone, heart, and other mesoderm cell types," Cell, vol. 166, no. 2, pp. 451-467, 2016.

[23] H. Sakurai, Y. Inami, Y. Tamamura, Y. Yoshikai, A. SeharaFujisawa, and K. Isobe, "Bidirectional induction toward paraxial mesodermal derivatives from mouse ES cells in chemically defined medium," Stem Cell Research, vol. 3, no. 2-3, pp. 157-169, 2009.

[24] Y. Naito, K. Hino, H. Bono, and K. Ui-Tei, "CRISPRdirect: software for designing CRISPR/Cas guide RNA with reduced off-target sites," Bioinformatics, vol. 31, no. 7, pp. 1120-1123, 2015.

[25] C. Yu, Y. Liu, T. Ma et al., "Small molecules enhance CRISPR genome editing in pluripotent stem cells," Cell Stem Cell, vol. 16, no. 2, pp. 142-147, 2015. 

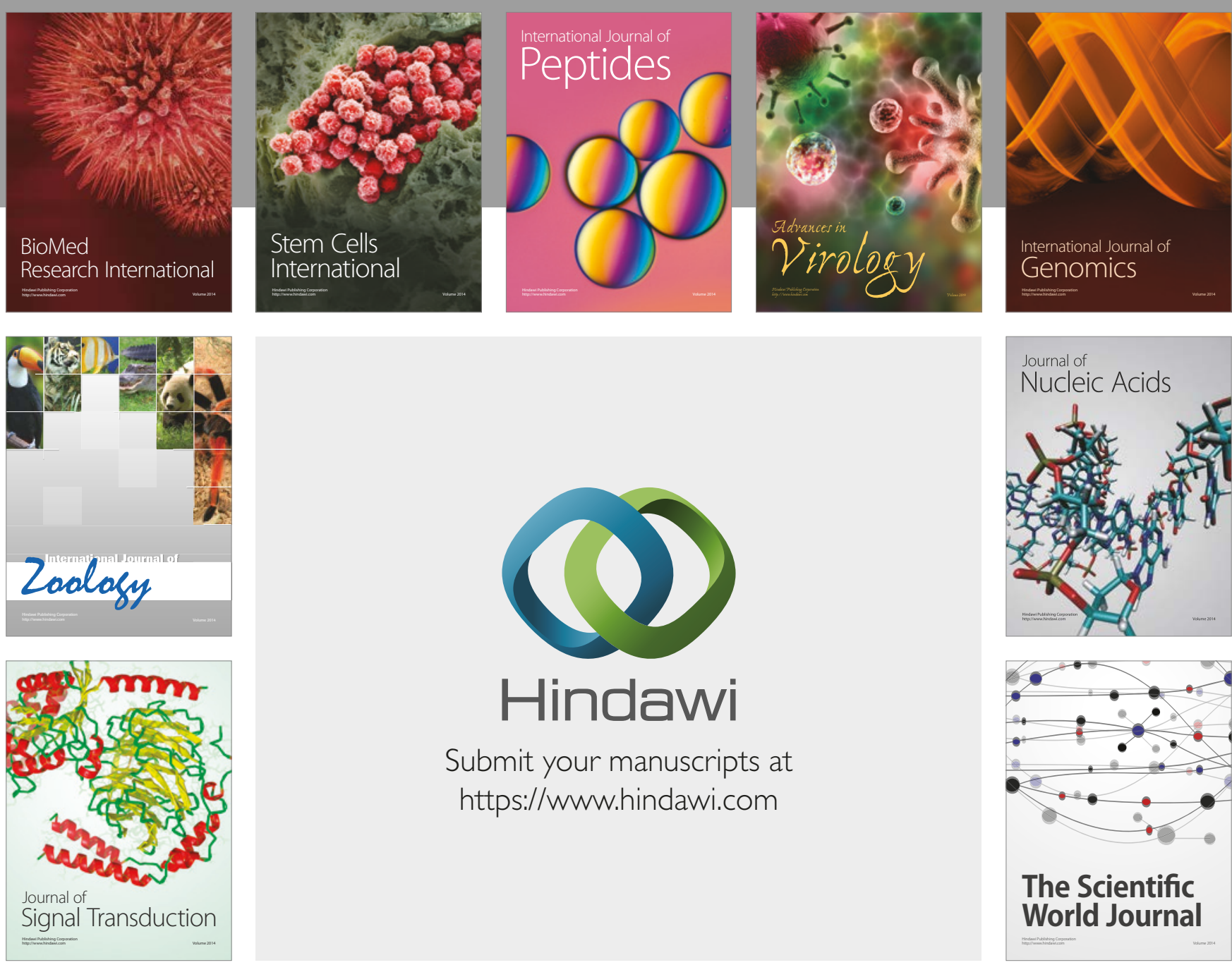

Submit your manuscripts at

https://www.hindawi.com
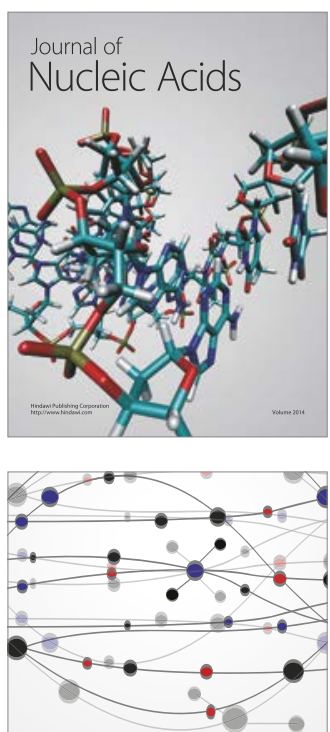

The Scientific World Journal

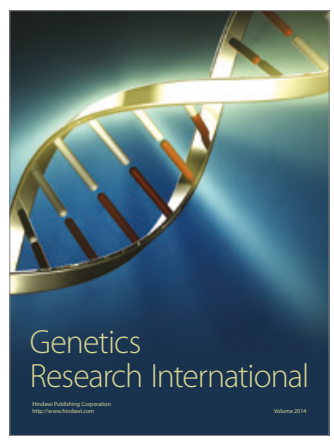

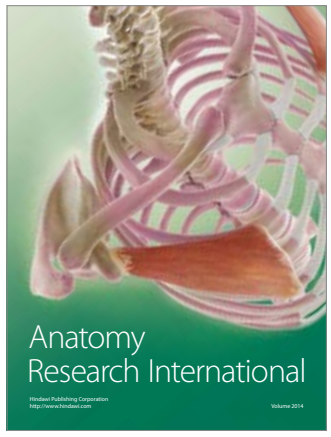

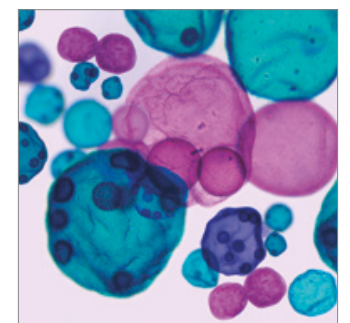

International Journal of Microbiology
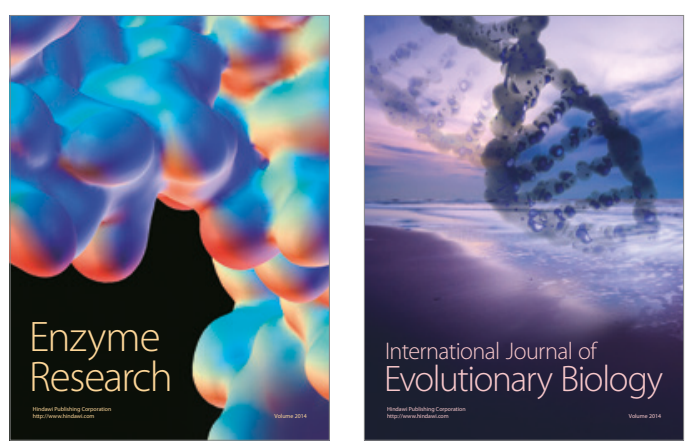
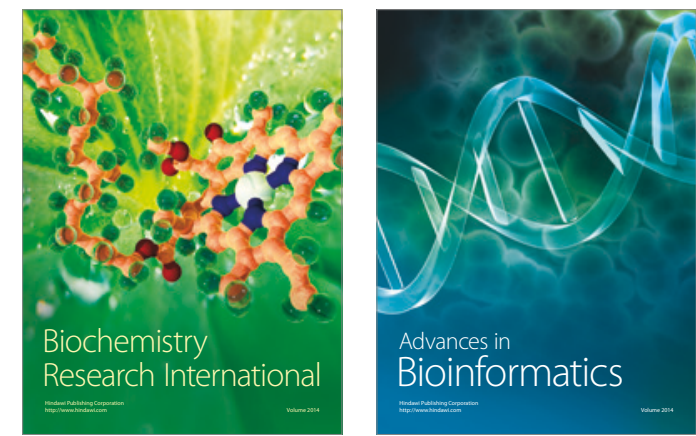

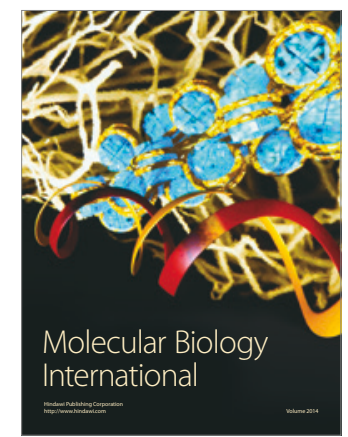

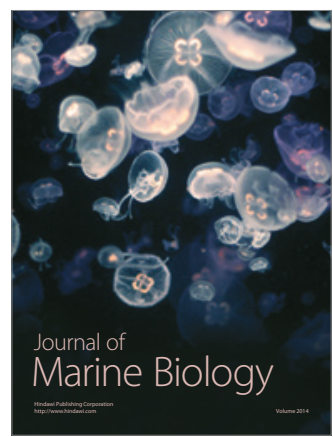

